\title{
Comparisons of temperament and character between problematic internet users and problematic drug users in Korean adolescents
}

\author{
Moon-Soo Lee*, In-Kwa Jung \\ Department of Neuropsychiatry, Korea University Medical Center, Korea University College of Medicine, Seoul, South Korea \\ Email: ${ }^{*}$ ppboard@gmail.com
}

Received 18 April 2012; revised 20 May 2012; accepted 30 May 2012

\begin{abstract}
Objective: It has been suggested that some internet users spend so much time on the internet that their personal and/or professional lives suffer. This study compared the temperament and character of problematic internet users (PIU) with that of problematic drug users (PDU) among Korean adolescents and aimed to examine the personality characteristics of adolescents with internet use problems. Method: We enrolled participants from high schools $(n=487)$, internet cafés $(n=89)$, and an adolescent-substance abuse consultation office $(n=45)$. All subjects were assessed using the Korean Internet Addiction Scale (KIAS), the Korean Adolescent Drug Addiction Screening Test-2 (KOADAST-2), and the Korean version of the Temperament and Character Inventory (K-TCI). Subjects were classified into the following four groups based on the KIAS and KOADAST-2 results: normal controls, pure PIUs, pure PDUs, and dual problematic users. Differences in the subscale scores on the K-TCI were analyzed by group. Results: Scores for Reward dependence (RD) were significantly lower in pure PIUs than in pure PDUs (13.85 \pm 4.07 versus $15.44 \pm 3.56, p<0.05)$. Scores for SelfDirectedness (SD) were lower in pure PIUs (18.21 \pm 6.90) and pure PDUs $(18.22 \pm 6.03)$ than in normal controls $(20.88 \pm 6.60)$, although the scores did not differ significantly between pure PIUs and pure PDUs. Scores for Cooperativeness (C) were lower in pure PIUs than in normal controls $(23.97 \pm 7.02$ versus $26.57 \pm 6.67, p<0.05)$. Conclusions: The results of this study suggest that PIUs are more socially insensitive and that they may experience more inter-personal problems than PDUs.
\end{abstract}

Keywords: Internet Addiction; Substance Abuse; Temperament; Interpersonal Relations

*Corresponding author.

\section{INTRODUCTION}

Internet addiction is characterized by a tenacity for activities associated with cyberspace, an excessive dependence on cyberspace activities, and the presence of mood changes, tolerance, withdrawal symptoms, and recurrence [1]. Internet addiction can be considered a behavioral addiction similar to that of TV addiction, video game addiction, and pathologic gambling [2].

Personality traits have been investigated using the Temperament and Character Inventory (TCI), which is based on Cloninger's theory. The TCI is a battery of tests designed to assess differences between people based on seven basic dimensions of temperament and character. Cloninger's biopsychosocial model of personality includes the psychological dimensions of temperament and character [3]. Cloninger first concentrated on the four biogenetic dimensions of temperament [4] and later extended his model to include three dimensions of character [3]. The four dimensions of temperament include novelty seeking (NS), harm avoidance (HA), reward dependence (RD), and persistence (PS), and the three character dimensions include self-directedness (SD), cooperativeness (C), and self-transcendence (ST).

Many studies have investigated the relationship between personality traits and various kinds of addiction [5, 6]. For example, numerous associations between personality traits and the use of tobacco, alcohol, and illegal drugs have been reported extensively. Novelty seeking, which is considered to be associated with low basal dopaminergic activity, has been reported to be associated with both nicotine consumption and heavy caffeine intake, both of which enhance dopaminergic neurotransmission [5]. Because personality may predispose, precipitate, or perpetuate substance addiction and/or dependence [7], information on personality traits might be useful in the management of addictive disorders and may assist clinicians in matching interventions to relevant personality traits [8]. 
The relationship between internet addiction and temperament has been assessed in previous studies. Ha et al. have reported that high harm avoidance, low self-directedness, low cooperativeness and high self-transcendence were correlated with internet addiction [9]. People with excessive internet use were also reported to show higher levels of harm avoidance behaviors and were thought to have genetic and personality traits similar to that of depressed patients [10]. In another study, people with risky internet use showed lower scores for RD, SD and C than normal internet users [11]. Cho et al. reported that problematic internet users had higher scores for the SD and $\mathrm{C}$ dimensions and lower scores for the NS and ST profiles of the Junior TCI version when compared with the non-problematic internet use group, suggesting that biogenetic temperament and character profile patterns may be one of the etiologies of problematic internet use [12]. Similarly, psychobiological traits were also assessed in online game users, and role playing game users were noted to have higher NS and SD scores. Role playing games were thought to be more attractive to latent game addicts with higher NS scores [13].

The TCI has also been used in many studies to better understand the personality traits and temperament of substance abusers and addicts [14,15]. Temperament and character personality profiles are known to be important in predicting and preventing addiction and can be applied in the treatment of such disorders [16].

There can be differences between internet addictions and other substance abuse. The negative aspects of substance abuse are well known. Society tries to control these behaviors by making them illegal. Substance abuse is a law violation and regarded as a risky and unaccepted behavior. However, the internet is not perceived by society as entirely negative, and normal use of the internet is not controlled by Korean culture. As a result, we postulate that substance abusers may start risk-taking behaviors more easily than internet addiction users. To elucidate these differences, we have conducted an analysis of the personality traits between internet users and drug users by using the TCI. A previous study in Taiwan has compared temperaments using a tridimensional personality questionnaire. In their results, high NS, high HA, and low RD were significant predictors for internet addiction. In contrast, high NS, low HA and low $\mathrm{RD}$ predicted a higher proportion of adolescents with substance use experience [17].

Investigating the personality traits of internet-addicted users and those of the substance abusers would help to characterize internet addiction. Drug addiction was selected for comparison with internet addiction since the personality traits of substance abusers are well known [5,7].

The aim of this study was to directly compare the temperament and character between problematic internet users (PIU) and problematic drug users (PDU) in Korean adolescents. The results of this study might elucidate the differences and similarities between internet addiction and drug addiction using measurements of temperament and character.

An understanding of the similarities and differences between these two groups may be helpful for both managing and preventing internet addiction.

\section{METHODS}

\subsection{Subjects and Procedures}

Adolescent participants aged 16 - 18 years of age were recruited at three sites; 1) a high school located in the southeast region of Seoul, South Korea, 2) internet cafés in Songpa-Gu, Seoul, South Korea, and 3) a YMCA adolescent substance abusers consultation office in Seocho-Gu, Seoul, South Korea. We selected these three sites to facilitate the enrollment of participants who had typical characteristics of each group (normal controls from high schools, PIUs from internet cafés, and PDUs from the substance-abuse consultation office). Demographic data including gender and age were collected, and subjects were rewarded for completing the questionnaires by receiving their survey results and small gifts. This study was approved by the institutional review board of the Korea University Medical Center. Each adolescent participated voluntarily and provided informed consent.

\subsection{Measures}

\subsubsection{Korean Internet Addiction Scale (KIAS) [18,19]}

This test was developed using psychometric techniques with support from the Korean government. This scale comprises 40 items that evaluate the following 7 impairment areas to screen for internet addiction: 1) disturbance of adaptive functions, 2) disturbance of reality testing, 3) addictive automatic thoughts, 4) withdrawal, 5) virtual interpersonal relationships, 6) deviant behavior, and 7) tolerance. This test can be used to classify respondents as high risk, potential risk, and normal users.

\subsubsection{Korean Adolescent Drug Addiction Screening Test-2 (KOADAST-2) [20,21]}

This test was developed to identify drug addiction among adolescents who have experiences of drug use and consists of 12 items that are each scored as $0,0.5$, or 1 . This test is a commonly used screen for drug addiction in Korea [22]. Total scores of 0.5 - 2.5 and 3 or more are considered indicative of drug abuse and drug dependence, respectively [20]; the absence of a history of drug use or problematic drug use yields a score of 0 . 


\subsubsection{Korean Version of TCI}

TCI is a self-reported instrument of yes/no answers that assess the following personality dimensions: NS (40 items), HA (35 items), RD (24 items), PS (eight items), SD (44 items), C (42 items), and ST (33 items). We used the Korean version of the TCI (K-TCI). The reliability and validity of the K-TCI has been confirmed previously [23].

\subsection{Analysis}

On the basis of the scores of the KIAS, all the partici- pants were classified into one of the following groups: high risk internet users, potential risk internet users, and normal internet users. We combined high risk and potential risk internet users into PIUs, resulting in the following two groups: PIUs and normal internet users. All participants were also classified into PDUs and non-drug users. Consequently, 4 groups (pure PIUs, pure PDUs, dual problematic users and normal controls) were identified for analysis by combining the results from the KIAS and KOADAST-2 (Figure 1). The mean scores of the scales were analyzed for high school students, internet
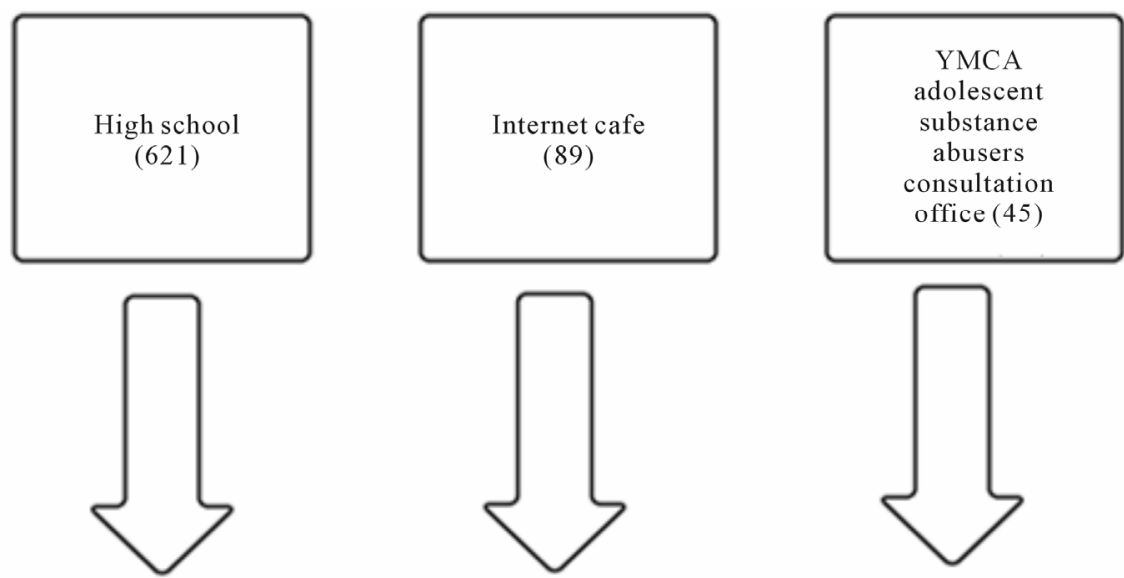

Korean Internet Addiction Scale (KIAS): problematic Internet user, normal Internet user

Korean Adolescent Drug Addiction Screening Test-2 (KOADAST-2)

problematic drug users, drug non-users

Korean version of temperament and character inventory

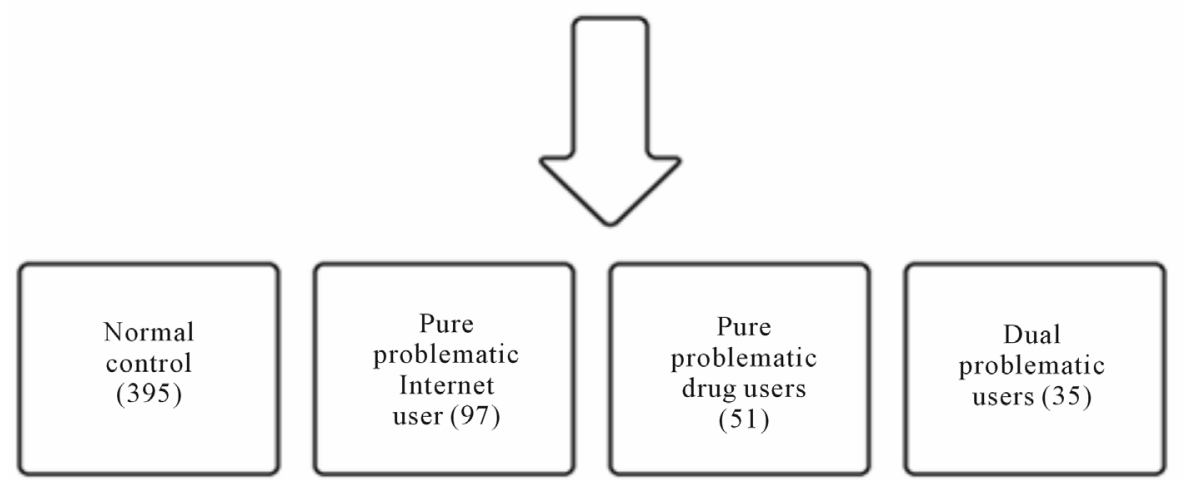

Figure 1. Classification of participants according to the results of KIAS and KOADAST-2. Numbers show the total number of participants. KIAS: Korean Internet Addiction Scale; KOADAST-2: Korean Adolescent Drug Addiction Screening Test-2. 
café users and substance abuse consultation office participants. Using an ANOVA with a post-hoc Tukey analysis, we compared the K-TCI scores among the 4 groups. A probability value of $\mathrm{p}<0.05$ was considered statistically significant. All data analyses were performed using SPSS $^{\circledR} 12.0$.

\section{RESULTS}

1) Characteristics of the subjects

A total of 621 subjects were enrolled in the study, including 487, 89, and 45 subjects from high schools, internet cafés, and substance-abuse consultation offices, respectively. The mean ages of the subjects were $16.77 \pm$ $0.46,16.62 \pm 1.06$, and $16.42 \pm 1.14$ years for the high schools students, internet café users, and substance-abuse consultation office participants, respectively. The gender distribution in each group is shown in Table 1. Significant differences were observed between males and females ( $\mathrm{p}<0.01$, chi-square test).

The distribution of PIUs differed significantly among the participant groups ( $p<0.05$, chi-square test). The distribution of the PDUs also differed significantly for each participant group ( $\mathrm{p}<0.01$, chi-square test) (Table 1).

2) KIAS and KOADAST-2 scores in subjects

We compared the mean KIAS and KOADAST-2 scores in the participant groups using ANOVA. The KIAS and KOADAST-2 scores were significantly higher ( $\mathrm{p}<$ $0.01)$ in internet cafe participants $(75.56 \pm 20.33$ and $1.28 \pm 2.55$, respectively) and substance-abuse consultation office participants $(74.20 \pm 27.35$ and $1.63 \pm 3.19$, respectively) than the high school students (64.58 \pm 17.60 and $0.31 \pm 1.25$, respectively). However, the scores on the two tests did not differ significantly between the internet cafe and substance-abuse consultation office participants (Table 2).

3) Temperament and characteristics in problematic internet and drug users

To clarify the differences in personality profiles between different types of addiction problems, we specifically compared scores on the K-TCI using an ANOVA with a post-hoc Tukey test between four groups: pure PIUs, pure PDUs, dual problematic users and normal controls. There were 395 (68.3\%) non-drug users, 97 (16.8\%) PIUs, 51 (8.8\%) PDUs, and 35 (6.1\%) dual problem users (Table 3). We found significant differences in the RD, SD, and C subscale scores (Table 4). The $\mathrm{RD}$ scores were lower in pure PIUs than in pure PDUs $(13.85 \pm 4.07$ versus $15.44 \pm 3.56, \mathrm{p}<0.05)$. The SD scores were lower in pure PIUs $(18.21 \pm 6.90)$ and pure PDUs $(18.22 \pm 6.03)$ than in normal controls, and the $\mathrm{C}$ scores were lower in pure PIUs than in normal

Table 1. Distributions of participant by gender and scores on the Korean Internet Addiction Scale (KIAS) and the Korean Adolescent Drug Addiction Screening Test-2 (KOADAST-2).

\begin{tabular}{|c|c|c|c|c|c|}
\hline & $\begin{array}{l}\text { High school students } \\
\qquad(\mathrm{n}=487)\end{array}$ & $\begin{array}{l}\text { Internet cafe users } \\
\qquad(\mathrm{n}=89)\end{array}$ & $\begin{array}{l}\text { Substance-abuse consultation } \\
\text { office participants }(n=45)\end{array}$ & $\chi^{2}$ & $\mathrm{p}$ \\
\hline Males & 247 (50.7\%) & $68(76.4 \%)$ & $40(88.9 \%)$ & & \\
\hline Females & $240(49.3 \%)$ & $21(23.6 \%)$ & $5(11.1 \%)$ & 40.214 & 0.001 \\
\hline Total & 487 (100\%) & $89(100 \%)$ & $45(100 \%)$ & & \\
\hline Problematic internet users & $100(20.5 \%)$ & $27(30.3 \%)$ & $14(31.1 \%)$ & 6.074 & 0.048 \\
\hline Total & 487 (100\%) & $89(100 \%)$ & $45(100 \%)$ & & \\
\hline Drug non-users & $406(88.5 \%)$ & $66(77.6 \%)$ & $27(60.0 \%)$ & & \\
\hline Problematic drug users & 53 (11.5\%) & 19 (22.4\%) & $18(40.0 \%)$ & 29.468 & 0.001 \\
\hline
\end{tabular}

Numbers differ from the total number of the participants $(n=621)$ because not all participants responded to every item on each questionnaire.

Table 2. Scores on the KIAS and KOADAST-2 for each participant group.

\begin{tabular}{ccccccc}
\hline & $\begin{array}{c}\text { High school } \\
(\mathrm{n}=487)\end{array}$ & $\begin{array}{c}\text { Internet cafe } \\
(\mathrm{n}=89)\end{array}$ & $\begin{array}{c}\text { Substance-abuse consultation office } \\
(\mathrm{n}=45)\end{array}$ & Total & $\mathrm{F}$ & $\mathrm{p}$ \\
\hline KIAS scores & $64.58 \pm 17.60^{\mathrm{ab}}$ & $75.56 \pm 20.33^{\mathrm{a}}$ & $74.20 \pm 27.35^{\mathrm{b}}$ & $66.87 \pm 19.34$ & 16.224 & 0.001 \\
KOADAST-2 scores & $0.31 \pm 1.25^{\mathrm{ab}}$ & $1.28 \pm 2.55^{\mathrm{a}}$ & $1.63 \pm 3.19^{\mathrm{b}}$ & $0.55 \pm 1.77$ & 21.206 & 0.001 \\
\hline
\end{tabular}

Same letters indicate $\mathrm{p}<0.05$ by Tukey's multiple comparisons. 
Table 3. Distribution of the subjects.

\begin{tabular}{|c|c|c|c|c|c|}
\hline & Drug non-users & Problematic drug users & Total & $\chi^{2}$ & $\mathrm{p}$ \\
\hline Problematic internet users & 97 (16.8\%) & 35 (6.1\%) & $132(22.8 \%)$ & & \\
\hline Normal internet users & 395 (68.3\%) & $51(8.8 \%)$ & 446 (77.2\%) & 18.289 & 0.001 \\
\hline Total & 492 (85.1\%) & 90 (14.9\%) & 578 (100.0\%) & & \\
\hline
\end{tabular}

Numbers differ from the total number of the participants $(n=621)$ because not all participants responded to every item on each questionnaire.

Table 4. Temperament and character scales for each comparison group.

\begin{tabular}{ccccccc}
\hline & $\begin{array}{c}\text { Normal controls } \\
(\mathrm{n}=395)\end{array}$ & $\begin{array}{c}\text { Pure problematic internet users } \\
(\mathrm{n}=97)\end{array}$ & $\begin{array}{c}\text { Pure problematic drug users } \\
(\mathrm{n}=51)\end{array}$ & $\begin{array}{c}\text { Dual problematic users } \\
(\mathrm{n}=35)\end{array}$ & $\mathrm{F}$ & $\mathrm{p}$ \\
\hline $\mathrm{NS}$ & $20.33 \pm 5.29$ & $20.59 \pm 4.84$ & $22.12 \pm 4.35$ & $21.00 \pm 3.92$ & 1.974 & 0.117 \\
$\mathrm{HA}$ & $17.56 \pm 6.49^{\mathrm{a}}$ & $19.55 \pm 6.28^{\mathrm{a}}$ & $17.49 \pm 5.99$ & $17.46 \pm 5.83$ & 2.657 & $0.048^{*}$ \\
$\mathrm{RD}$ & $14.87 \pm 3.98^{\mathrm{a}}$ & $13.68 \pm 4.01^{\mathrm{a}}$ & $15.35 \pm 3.56$ & $13.54 \pm 3.58$ & 3.841 & $0.010^{*}$ \\
P & $4.43 \pm 1.95$ & $4.10 \pm 1.85$ & $4.22 \pm 1.70$ & $3.86 \pm 1.70$ & 1.626 & 0.182 \\
SD & $20.87 \pm 6.61^{\mathrm{ab}}$ & $17.97 \pm 6.58^{\mathrm{a}}$ & $18.49 \pm 6.13^{\mathrm{b}}$ & $19.46 \pm 6.84$ & 6.308 & $0.000^{* *}$ \\
C & $26.54 \pm 6.65^{\mathrm{ab}}$ & $23.81 \pm 6.97^{\mathrm{a}}$ & $25.35 \pm 7.69$ & $23.34 \pm 5.31^{\mathrm{b}}$ & 6.044 & $0.000^{* *}$ \\
ST & $14.76 \pm 5.70$ & $15.90 \pm 5.53$ & $16.02 \pm 4.92$ & $16.69 \pm 4.79$ & 2.537 & 0.056 \\
\hline
\end{tabular}

NS: novelty seeking; HA: harm avoidance; RD: reward dependence; P: persistence; SD: self-directedness; C: cooperativeness; ST: self-transcendence; ${ }^{*}$ p < 0.05; ${ }^{* *} \mathrm{p}<0.01$. Same letters indicate $\mathrm{p}<0.05$ by Tukey's multiple comparisons.

controls $(23.97 \pm 7.02$ versus $26.57 \pm 6.67, \mathrm{p}<0.05)$. However, the SD scores did not differ between pure PIUs and pure PDUs. The NS and P scores did not significantly differ among these four groups.

\section{DISCUSSION}

On the basis of the internet addiction test, we classified each participant as a PIU or a normal internet user. PIUs and normal internet users comprised $20.5 \%$ and $79.5 \%$ of the high school students, respectively. These rates are similar to those reported in a previous study [19]. The proportions of PIUs were higher in the internet café and substance-abuse consultation office groups than in the high school student group. This result was to be expected because we specifically selected these two sites (internet café and substance-abuse consultation office) for recruiting adolescents who had addiction problems.

NS represents a dopaminergically modulated and heritable tendency toward frequent exploratory activity and intense excitement in response to novelty [3]. High NS is reportedly associated with substance abuse by adolescents [24]. All three groups (pure problematic internet users, pure problematic drug users, and dual problematic users) also showed higher mean NS scores than normal controls in this study, although this difference was not statistically significant.

Individuals high in HA tend to be cautious, careful, fearful, and tense. In this study, pure PIUs showed higher
HA scores than normal controls. This result corresponds to previous reports [17] and assumptions that pure PIUs tend to avoid risk-taking behaviors when negative results are expected.

The RD subscale scores differed significantly between pure PIUs and pure PDUs. RD involves maintaining or continuing behaviors that have been previously associated with reinforcement and is manifested by sensitivity, sentimentality, and dependency on the approval of others [3]. Individuals with low RD typically exhibit a tendency toward social detachment.

In the post-hoc analysis, the SD scores were significantly lower in both pure PIUs and pure PDUs than in normal controls. People who have lower SD scores tend to feel asthenic and commonly appear weak and fragile to others. Their behaviors tend to be dominated by reactions to external stimuli rather than by their own personal goals. Their willpower appears to be too weak to overcome strong temptations. Low SD scores in other groups of substance abusers have been reported in previous studies [14].

Scores for the cooperative dimensions of the assessment were also lower in pure PIUs than in normal controls. Subjects with lower C scores tend to be more selfabsorbed, prefer solitude, and have poor social skills. The PIU groups showed significantly lower scores on the cooperation subscale than normal controls. They also showed lower scores than the PDUs, although this difference was not statistically significant in the post-hoc 
analysis. Participants with lower $\mathrm{C}$ scores tend to be more self-absorbed, prefer solitude, and have poor social relationships with peers. This finding suggests that there are differences in the quality of interpersonal relationships between problematic internet and drug users. Similar social skill impairment in frequent internet users has also been reported in another study [25]. The findings of the $\mathrm{RD}$ and $\mathrm{C}$ scores, which were lower in pure PIUs, suggest that differences exist in the quality of interpersonal relationships between pure PIUs and pure PDUs.

Problematic internet use can be regarded as another form of behavioral addiction. Therefore, we hypothesized that PIUs share common temperamental characteristics with PDUs in the TCI evaluation. This study also showed that although these two kinds of addictive patterns have common temperamental characteristics, they are not the same and might be different from each other with respect to sociability and interpersonal relationships.

There are several limitations to this study, which should be taken into consideration when interpreting the results. First, the inclusion criteria were determined by associates working at the recruitment sites and not by professionals who have a specialty in addictions. We did not use formal diagnostic criteria, such as the Diagnostic and Statistical Manual of Mental Disorders-IV (DSMIV), for the clinical diagnosis of PDUs. Second, the number of subjects in the PDU group was relatively small. Given the limited sample size, the differences in TCI subscale scores for PDUs were not statistically significant. However, they showed higher NS and lower HA than the other two groups. This pattern is consistent with previous reports [6,17].

Pure PIUs seem to experience more social insensitiveness and interpersonal problems than adolescents who do not have problematic internet use patterns. Further research is required to develop management programs targeted at these social and interpersonal characteristics.

\section{REFERENCES}

[1] Brian, D.N. and Wiemer-Hastings, P. (2005) Addiction to the internet and online gaming. CyberPsychology and Behavior, 8, 110-113. doi:10.1089/cpb.2005.8.110

[2] Holden, C. (2001) "Behavioral” addictions: Do they exist? Science, 294, 980-982. doi:10.1126/science.294.5544.980

[3] Cloninger, C.R., Przybeck, T.R., Svrakic, D.M. and Wetzel, R.D. (1994) The temperament and character inventory (TCI): A guide to its development and use. Center for Psychobiology of Personality, Washington University, St. Louis.

[4] Cloninger, C.R. (1987) A systematic method for clinical description and classification of personality variants. A proposal. Archives of General Psychiatry, 44, 573-588. doi:10.1001/archpsyc.1987.01800180093014

[5] Gurpegui, M., Jurado, D., Luna, J.D., Fernandez-Molina, C., Moreno-Abril, O. and Galvez, R. (2007) Personality traits associated with caffeine intake and smoking. Progress in Neuro-Psychopharmacology \& Biological Psychiatryis, 31, 997-1005. doi:10.1016/j.pnpbp.2007.02.006

[6] Wills, T.A., Vaccaro, D. and McNamara, G. (1994) Novelty seeking, risk taking, and related constructs as predictors of adolescent substance use: An application of Cloninger's theory. Journal of Substance Abuse, 6, 1-20. doi:10.1016/S0899-3289(94)90039-6

[7] Le Bon, O., Basiaux, P., Streel, E., Tecco, J., Hanak, C., Hansenne, M., et al. (2004) Personality profile and drug of choice; a multivariate analysis using Cloninger's TCI on heroin addicts, alcoholics, and a random population group. Drug and Alcohol Dependence, 73, 175-182. doi:10.1016/j.drugalcdep.2003.10.006

[8] Staiger, P.K., Kambouropoulos, N. and Dawe, S. (2007) Should personality traits be considered when refining substance misuse treatment programs? Drug and Alcohol Review, 26, 17-23. doi:10.1080/09595230601036952

[9] Ha, J.H., Kim, S.Y., Bae, S.C., Bae, S., Kim, H., Sim, M., et al. (2007) Depression and internet addiction in adolescents. Psychopathology, 40, 424-430. doi:10.1159/000107426

[10] Lee, Y.S., Han, D.H., Yang, K.C., Daniels, M.A., Na, C., Kee, B.S., et al. (2008) Depression like characteristics of 5HTTLPR polymorphism and temperament in excessive internet users. Journal of Affective Disorders, 109, 165169. doi:10.1016/j.jad.2007.10.020

[11] Lee, M.-S., Hong, S.-D. and Joung, Y.-S. (2007) Characteristics of temperament and character properties in adolescents with internet addiction tendency. Journal of Korean Neuropsychiatric Association, 46, 65-70. doi:10.5124/jkma.2007.50.1.65

[12] Cho, S.C., Kim, J.W., Kim, B.N., Lee, J.H. and Kim, E.H. (2008) Biogenetic temperament and character profiles and attention deficit hyperactivity disorder symptoms in Korean adolescents with problematic Internet use. CyberPsychology and Behavior, 11, 735-737. doi:10.1089/cpb.2007.0285

[13] Kim, J.W., Han, D.H., Park, D.B., Min, K.J., Na, C., Won, S.K., et al. (2010) The relationships between online game player biogenetic traits, playing time, and the genre of the game being played. Psychiatry Investigation, 7, 17-23.

[14] Hosak, L., Preiss, M., Halir, M., Cermakova, E. and Csemy, L. (2004) Temperament and character inventory (TCI) personality profile in metamphetamine abusers: A controlled study. European Psychiatry, 19, 193-195. doi:10.1016/j.eurpsy.2004.04.003

[15] Chakroun, N., Doron, J. and Swendsen, J. (2004) Substance use, affective problems and personality traits: Test of two association models. Encephale, 30, 564-569. doi:10.1016/S0013-7006(04)95471-1

[16] Schinka, J.A., Curtiss, G. and Mulloy, J.M. (1994) Personality variables and self-medication in substance abuse. 
Journal of Personality Assessment, 63, 413-422. doi:10.1207/s15327752jpa6303 2

[17] Ko, C.H., Yen, J.Y., Chen, C.C., Chen, S.H., Wu, K. and Yen, C.F. (2006) Tridimensional personality of adolescents with internet addiction and substance use experience. Canadian Journal of Psychiatry, 51, 887-894.

[18] Kim, C. and Lee, S. (2004) Development and Validation study on internet addiction-proneness scale. 112th Annual Convention of American Psychological Association, Honolulu, Hawaii.

[19] Kim, C.T., Kim, D.I., Park, J.K. and Lee, S.J. (2002) A study on internet addiction counseling and the development of prevention programs. Korean Agency for Digital Opportunity and Promotion.

[20] Kim, K.-B. (1997) A study of early detection of adolescent drug user's and adolescent drug addicts. Korean Journal of Youth Studies, 4, 209-223.

[21] Kim, K.-B., Lee, C.-K. and Kim, B.-S. (1995) The preliminary study of the Korean Adolescent Drug Addiction Screening Test (KOADAST) (II). Journal of Korean
Neuropsychiatric Association, 34, 1862-1874.

[22] Kwon, M.Y. (1998) The individual, familial, and peerrelational characteristics of drug-abused adolescent. Graduate School of Kyungpook National University, Taegu.

[23] Sung, S.M., Kim, J.H., Yang, E., Abrams, K.Y. and Lyoo, I.K. (2002) Reliability and validity of the Korean version of the temperament and character inventory. Comprehensive Psychiatry, 43, 235-243. doi:10.1053/comp.2002.30794

[24] Laucht, M., Becker, K., Blomeyer, D. and Schmidt, M.H. (2007) Novelty seeking involved in mediating the association between the dopamine D4 receptor gene exon III polymorphism and heavy drinking in male adolescents: Results from a high-risk community sample. Biological Psychiatry, 61, 87-92. doi:10.1016/j.biopsych.2006.05.025

[25] Engelberg, E. and Sjoberg, L. (2004) Internet use, social skills, and adjustment. CyberPsychology and Behavior, 7, 41-47. doi:10.1089/109493104322820101 